\title{
Scattering properties and femtosecond laser ablation thresholds of human and canine vocal folds at 776-nm wavelength
}

Liam Andrus

Ted Mau

Adela Ben-Yakar 


\title{
Scattering properties and femtosecond laser ablation thresholds of human and canine vocal folds at 776-nm wavelength
}

\author{
Liam Andrus, ${ }^{a}$ Ted Mau, ${ }^{b}$ and Adela Ben-Yakar ${ }^{a, c, *}$ \\ ${ }^{a}$ University of Texas at Austin, Department of Biomedical Engineering, Austin, Texas, United States \\ bUniversity of Texas Southwestern Medical Center, Department of Otolaryngology-Head and Neck Surgery, Dallas, Texas, United States \\ 'University of Texas at Austin, Department of Mechanical Engineering, Austin, Texas, United States
}

\begin{abstract}
Ultrafast laser ablation may provide a treatment for vocal fold (VF) scarring. Optical properties of VFs must be known prior to clinical implementation to select appropriate laser surgery conditions. We present scattering lengths of epithelium $\ell_{s, \mathrm{ep}}$, superficial lamina propria $\ell_{\mathrm{s}, \mathrm{SLP}}$, and ablation thresholds $F_{\text {th }}$ of human and canine VF tissues. Our experimental approach involves an image-guided, laser-ablation-based method that allows for simultaneous determination of $\ell_{s}$ and $F_{\text {th }}$ in these multilayered tissues. Studying eight canine samples, we found $\ell_{\mathrm{s}, \mathrm{ep}}=75.3 \pm 5.7 \mu \mathrm{m}, \ell_{\mathrm{s}, \mathrm{SLP}}=26.1 \pm 1.2 \mu \mathrm{m}, F_{\text {th,ep }}=1.58 \pm 0.06 \mathrm{~J} / \mathrm{cm}^{2}$, and $F_{\text {th,SLP }}=1.55 \pm 0.17 \mathrm{~J} / \mathrm{cm}^{2}$. Studying five human samples, we found $\ell_{\mathrm{s} \text {,ep }}=42.8 \pm 3.3 \mu \mathrm{m}$ and $F_{\text {th,ep }}=$ $1.66 \pm 0.10 \mathrm{~J} / \mathrm{cm}^{2}$. We studied the effects of cumulative pulse overlap on ablation threshold and found no significant variations beyond 12 overlapping pulses. Interestingly, our studies about the effect of sample storage on the scattering properties of porcine VF show a $60 \%$ increase in $\ell_{s, e p}$ for fresh porcine VF when compared to the same sample stored in isotonic solution. These results provide guidelines for clinical implementation by enabling selection of optimal laser surgery parameters for subsurface ablation of VF tissues. $\odot$ The Authors. Published by SPIE under a Creative Commons Attribution 4.0 Unported License. Distribution or reproduction of this work in whole or in part requires full attribution of the original publication, including its DOI. [DOI: 10.1117/1.JBO.24.8.085005]
\end{abstract}

Keywords: scattering measurements; tissue ablation, ultrafast lasers; ultrafast laser surgery; vocal folds; vocal folds scarring.

Paper 190162R received May 24, 2019; accepted for publication Aug. 14, 2019; published online Aug. 30, 2019.

\section{Introduction}

Vocal fold (VF) scarring is a primary cause of voice disorders, which affect an estimated 2 to 6 million people in the U.S. alone. ${ }^{1-3}$ Scar tissue forms as an inflammatory response to external trauma, hindering phonation by reducing viscoelasticity of the vibratory layers of the VF. ${ }^{4,5}$ The lamina propria (LP), a subepithelial tissue layer consisting primarily of collagen, elastin, and reticulin fibers, is largely responsible for the vibratory response associated with normal phonation and thus is highly sensitive to scar formation. Current research focuses on restoration therapies for the superficial lamina propria (SLP) including development of various injectable therapeutic biomaterials that could promote a more favorable reparative response. ${ }^{6-10}$ These therapies would benefit from the creation of subepithelial voids within the scarred tissue region of interest (ROI), allowing for a "pocket" to localize injected biomaterials for an extended period. Preclinical studies, demonstrating that injection into stiff scar tissue results in poor biomaterial localization and treatment repeatability, highlight the potential therapeutic benefit of subepithelial void formation. ${ }^{11-13}$

One possible solution utilizes ultrafast laser ablation to create subepithelial voids within the SLP. ${ }^{14,15}$ The nonlinear dependence of multiphoton absorption on intensity confines energy deposition to femtoliter sized focal volumes if appropriately high numerical aperture (NA) focusing optics are used. In addition, creation of deep voids becomes feasible when using wavelengths within the optical window of tissues

*Address all correspondence to Adela Ben-Yakar, E-mail: ben-yakar@mail .utexas.edu $(\lambda \approx 700-1400 \mathrm{~nm})$, allowing for bulk material dissection without disturbing superficial tissue layers. Prior work by our group successfully demonstrated creation of voids $\sim 100 \mu \mathrm{m}$ beneath the tissue surface, well within the SLP of porcine VF with an epithelium thickness of 50 to $80 \mu \mathrm{m} .^{14,16}$

Optical scattering properties of VF tissue must be known before implementing subsurface void formation for biomaterial injection in human patients. The literature only presents a small number of studies discussing the optical properties of human VFs. Among these studies, Mahlstedt et al. ${ }^{17}$ utilized a double integrating sphere with Monte Carlo simulations to measure scattering and absorption coefficients over the 400- to 2200-nm wavelength range. Their results indicate a scattering length $\ell_{s}=$ $83 \mu \mathrm{m}$ at $\lambda=700 \mathrm{~nm}$ for bulk human VF. Since they homogenized their samples in a precooled mortar prior to measurements, they did not have the ability to differentiate between optical properties of the epithelium and SLP. Additionally, homogenization is known to decrease optical scattering, thus reducing similarity to native tissue. ${ }^{18}$ Prior work by our group and others has shown variations in optical properties for different tissue layers, ${ }^{16,19-21}$ and a priori knowledge of the optical properties of individual tissue layers is required to implement subsurface void creation for the proposed scarred VF treatment method.

In this paper, we present scattering properties for different tissue layers of freshly excised human and canine VFs and study how tissue storage conditions in isotonic solution can vary these properties. We also present ablation thresholds to create voids below the tissue surface and study how the number of overlapping laser pulses can affect these ablation thresholds. To overcome limitations associated with current tissue scattering 
measurement methods and enable assessment of multilayered tissues, we use a method based on ultrafast laser ablation. This image-guided method, which we proposed in Ref. 16 and expanded in Ref. 19, allows for simultaneous determination of scattering lengths and ablation thresholds of individual layers in thick, multilayer tissues without requiring any sample processing. Results, obtained from multiple human VF samples as well as canine, which is the animal model of choice for preclinical testing of laryngeal treatments, provide a guideline for designing the necessary laser surgery conditions for future clinical treatment.

\section{Experimental Approach}

To measure the optical properties of excised human and canine VF samples, we used a laser ablation-based method that we recently developed. ${ }^{19}$ Using this method, we can simultaneously determine both scattering lengths and ablation thresholds in multiple layers of a heterogenous tissue. The method relies on finding input energies required to initiate ablation at multiple depths in each tissue layer. Assuming only ballistic photons contribute to nonlinear ablation, the threshold fluence at the focal plane of a single-layered tissue can be described by Beer's law:

$F_{\text {th }}=\frac{E_{\mathrm{th}, \mathrm{surf}}}{\pi w_{o}^{2}} e^{-\frac{z_{\mathrm{ab}}}{t_{s}}}$,

where $E_{\text {th,surf }}$ is the input threshold energy at the tissue surface, $w_{0}$ is the $1 / e^{2}$ beam radius, $z_{\mathrm{ab}}$ is the ablation depth in tissue, and $\ell_{s}$ is a direct measure (i.e., not reduced) of the scattering length. If $E_{\mathrm{th} \text {,surf }}$ is found at two depths, one can then determine the scattering length according to

$\ell_{s}=\frac{z_{\mathrm{ab}, 2}-z_{\mathrm{ab}, 1}}{\ln \left(E_{\mathrm{th}, \text { surf }, 2}-E_{\mathrm{th}, \text { surf }, 1}\right)}$.

One can determine threshold fluence $F_{\text {th }}$ by inserting $\ell_{s}$ into Eq. (1). For multilayered tissues, Eq. (2) can be used to solve for $\ell_{s}$ if each tissue layer is considered as a distinct, homogeneous medium. Threshold fluences in deep tissue layers can be found by extending Eq. (1) to consider the properties of multiple superficial layers, in our case epithelium and SLP:

$F_{\mathrm{th}}=\frac{E_{\mathrm{th}, \mathrm{surf}}}{\pi w_{o}^{2}} e^{-\left(\frac{z_{\mathrm{ep}}}{t_{s, \mathrm{ep}}}+\frac{z_{\mathrm{ab}}-z_{\mathrm{ep}}}{t_{s, \mathrm{SLP}}}\right)}$,

where $z_{\mathrm{ep}}$ and $\ell_{s \text {,ep }}$ are the thickness and scattering length of the epithelium, and $\ell_{s, \mathrm{SLP}}$ is the SLP scattering length. For homogenous media, damage is expected to occur at a sharp threshold, as is the case for most metals and glass. ${ }^{22,23}$ However, this assumption does not hold in tissue, as heterogeneity in tissue scatterer distribution may lead to a broad range of energies over which ablation occurs. To address the effects of tissue heterogeneity on threshold determination, $E_{\mathrm{th}, \text { surf }}$ is found by recording percent ablation in a given field of view (FOV) for a range of pulse energies near threshold. The pulse energy required for $50 \%$ FOV ablation is found by fitting an error function to the collected data:

percent ablation $=50\left[1+\operatorname{erf}\left(\frac{E-E_{\text {th,surf }}}{1.05 \Delta E}\right)\right]$,

where $\Delta E$ is the difference in pulse energies required to reach $25 \%$ and $75 \%$ FOV ablation. If $E_{\text {th.surf }}$ is found at multiple depths in each tissue layer, a nonlinear least squares fit of the natural $\log$ of $E_{\mathrm{th} \text {,surf }}$ versus ablation depth allows for determination of $\ell_{s}$, defined as the inverse slope [according to Eq. (2)] and $F_{\text {th }}$ [according to Eq. (3)].

The experimental setup includes a home-made, upright twophoton microscope that employs an Er-doped fiber laser (1.0 ps pulse width, frequency doubled to $\lambda=776 \mathrm{~nm}$, Discovery, Raydiance Inc.) for both imaging and ablation. We use a repetition rate (RR) of $303 \mathrm{kHz}$ for imaging and ablation during experiments. A pair of galvanometric mirrors raster scans the incoming laser beam, which is expanded onto the back aperture of a coverslip-corrected air objective (20×, NA 0.75, Plan Apo, Nikon) and focused on the tissue. Two-photon autofluorescence is epi-collected, filtered (Schott BG39), and detected by a photomultiplier tube (PMT). The galvanometric mirrors, PMT, and stages are controlled by MPScan software suite. ${ }^{24}$

To determine the beam waist at the focal plane inside scattering media, we imaged 100-nm fluorescent beads (FluoSpheres ${ }^{\mathrm{TM}}$, $2 \%$ solids, Invitrogen ${ }^{\mathrm{TM}}$ ), suspended in $2 \%$ low-melt-point agar gel. We added $0.95-\mu \mathrm{m}$ polystyrene beads (Bangs Lab) to the suspension at a concentration of $1.85 \times 10^{10}$ beads $/ \mathrm{mL}$ to provide an estimated $\ell_{s} \approx 46 \mu \mathrm{m}$, representing the expected scattering length of tissue. Bead solutions were added to agar, vortexed, set within a coverslip mold with spacers of known thickness, and allowed to solidify. Analysis of two-photon images at multiple depths determined $1 / e^{2}$ beam waste radius $w_{0}=0.75 \pm$ $0.01 \mu \mathrm{m}$. This value was consistent over 10 depths in the 0 to $96-\mu \mathrm{m}$ range, indicating minimal change in spot size due to spherical aberrations and scattering.

We tested eight canine and five human VF samples. Canine VFs were harvested immediately following euthanization for unrelated research projects. Human VF samples were collected under an Institutional Review Board (UT Southwestern)approved tissue collection protocol. The tissue was excised from VFs unaffected by tumor in total laryngectomy specimens immediately after the larynx was removed in surgery. For both canine and human samples, we excised the specimen for testing as a rectangular piece of VF mucosa from the mid-VF by carefully dissecting the mucosa off the underlying muscle. The mucosal specimens were placed in phosphate-buffered saline (PBS) on ice and overnight shipped to UT Austin. The samples were kept at $4^{\circ} \mathrm{C}$ until testing, which typically took place within 24 to $48 \mathrm{~h}$ of tissue harvest. For measurements, we set the VFs in a Petri dish with $170-\mu \mathrm{m}$ glass coverslip and submerged them in PBS to prevent desiccation. Fluorescent beads deposited onto the tissue surface aided in detection of the epithelium. Prior to ablation experiments, we determined the epithelial thickness by obtaining $z$-stacks in three different areas adjacent to the ROI. The RR of the laser was changed from $303 \mathrm{kHz}$ to $2 \mathrm{MHz}$ during $z$-stack image acquisition to acquire higher quality images before starting the experiments to determine average epithelium thickness with high certainty.

To measure optical properties of VF samples, we ablated a $40 \times 40 \mu \mathrm{m}^{2}$ FOV in a single frame by scanning 512 lines at 1.11 frames per second (fps), resulting in $N \approx 301$ pulse overlap. We found the number of spots available in our ablation FOV by dividing the FOV size $\left(40 \times 40 \mu \mathrm{m}^{2}\right)$ by our beam area $\left(\pi w_{0}^{2}\right)$ and used this with the RR of the laser to calculate $N$ according to the following:

$N=\frac{\mathrm{RR}}{\mathrm{fps}} \times \frac{\pi w_{0}^{2}}{\mathrm{FOV}}$. 
We acquired images over a slightly larger FOV of $80 \times 80 \mu \mathrm{m}^{2}$ at $1.11 \mathrm{fps}$ to cover the entire pre- and postablated areas to determine the size of the ablated region. The pulse energy required for $50 \%$ ablation $E_{\text {th,surf }}$ was found at eight depths per sample: four depths in the epithelium and four depths in the SLP. Ablation curves were generated at each depth by titrating over a range of pulse energies and recording the percent of FOV ablated at each energy. We acquired images as the average of 10 images and performed smoothing using a Gaussian filter (window size $10 \times 10$ ) prior to binarization. We utilized Otsu's method to threshold before and after images and applied the same threshold to each image pair to calculate ablation percentage as the ratio of pixels below threshold after ablation to pixels below threshold before ablation within FOV.

\section{Results and Discussion}

\subsection{Human and Canine Vocal Fold Scattering Length and Ablation Threshold Study}

Example two-photon autofluorescence images, taken before and immediately following ablation in canine VF \#1, are shown in Fig. 1(a) for a range of pulse energies at a depth of $36 \mu \mathrm{m}$. Only one depth in a single specimen is displayed for brevity. We could observe in all two-photon images a smooth transition from cellrich epithelium to LP with randomly organized collagen fibers, which are seen in normal SLP. ${ }^{16}$
Data shown in Fig. 1(c) represents pulse energies and corresponding FOV ablation percentage at $z_{\mathrm{ep}}=36 \mu \mathrm{m}$ in canine VF \#1. Collected data were fit according to Eq. (4), and the dotted red line indicates the point of $50 \%$ ablation where we defined $E_{\text {th,surf }}=43.9 \pm 1.5 \mathrm{~nJ}$ with $\Delta E=5.5 \pm 1.6 \mathrm{~nJ}$. Although curves similar to Fig. 1(c) were generated for every depth in each tissue sample, a single dataset is displayed for brevity.

We repeated this procedure to determine $E_{\text {th,surf }}$ at the seven remaining depths on the VF of canine \#1. Figure 1(d) shows the semilogarithmic plot of $E_{\text {th,surf }}$ versus ablation depth from which $\ell_{s}$ was deduced according to Eq. (2) and $F_{\text {th,ep }}$ and $F_{\text {th,SLP }}$ were then found using Eqs. (1) and (3), respectively. The process was repeated for the seven remaining canine tissues and five human specimens. Results are tallied in Table 1.

Uncertainty in individual $\ell_{s, \mathrm{ep}}$ and $\ell_{s, \mathrm{SLP}}$ measurements represents the standard error in fitting parameters for a weighted nonlinear least squares estimation of parameters. Weights were taken as the inverse of the variance for each approximated $E_{\text {th,surf }}$ value. Uncertainty in $E_{\text {th,surf }}$ and beam waist $\left(w_{0}\right)$ measurements were propagated forward to determine uncertainties presented for $F_{\text {th,ep }}$, and uncertainty in $F_{\text {th,SLP }}$ was estimated using uncertainty propagation to account for uncertainty in $z_{\mathrm{ep}}, \ell_{s, \mathrm{ep}}, \ell_{s, \mathrm{SLP}}$, and $E_{\mathrm{th}, \text { surf }}$. Uncertainty of averaged values represents the root-mean-square error of contributing data points.

We determined epithelium thickness $z_{\text {ep }}$ by measuring the depth of transition from the cell-rich epithelium to the densely (a)
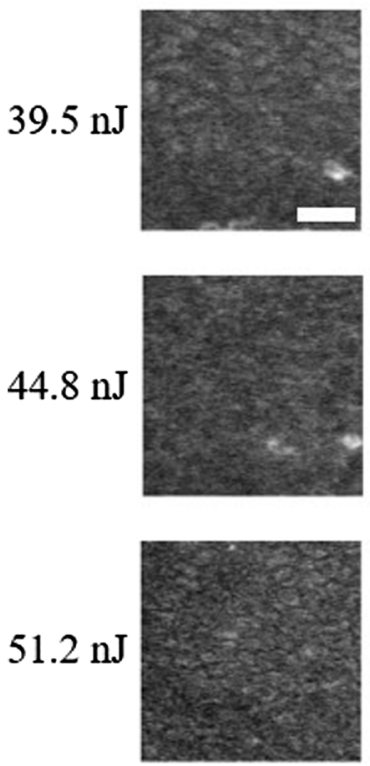

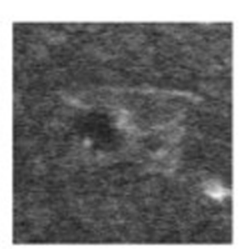

(b)
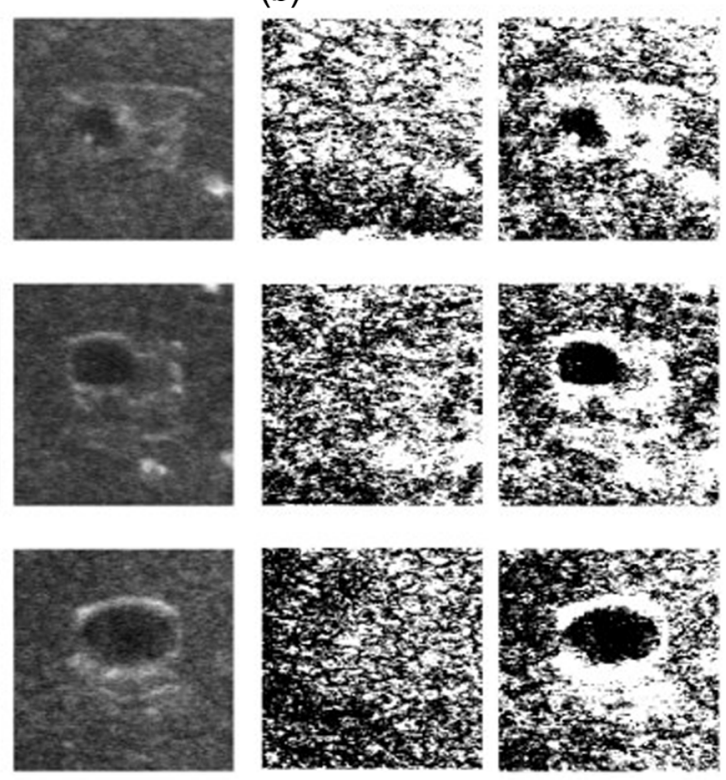

(c)

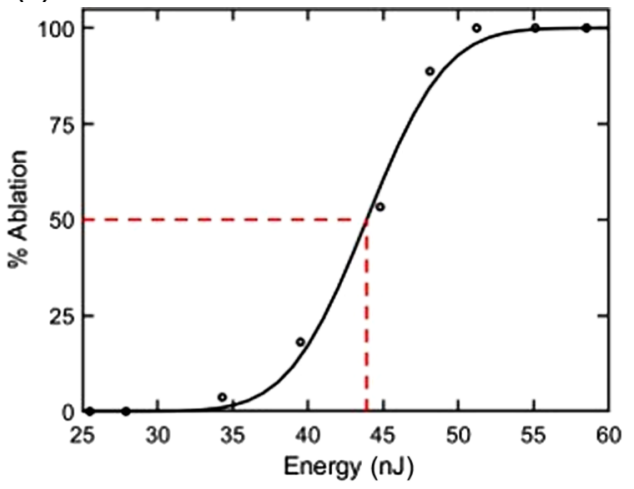

(d)

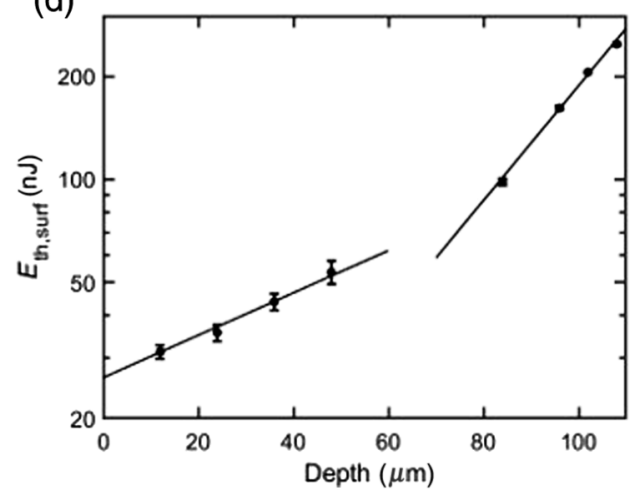

Fig. 1 Example data used in the scattering length and fluence threshold measurements. (a) Example two-photon images of $36 \mu \mathrm{m}$ depth for canine VF sample \#1. Left and right columns are images taken before and after ablation at the listed pulse energies. (b) Binarization of images in (a) to allow for percent FOV ablation according to Eq. (4). Scale bar represents $20 \mu \mathrm{m}$. (c) Ablation curve for experiments presented in (a); the dotted line specifies the point of $50 \%$ ablation $E_{\text {th }}$. (d) Ablation depth versus $E_{\text {th,surf }}$ plotted as a semilogarithmic for clarity. The SLP begins around $60-\mu \mathrm{m}$ depth, marked by a change in slope for the deeper layer. Errors bars represent $95 \%$ confidence intervals for the fit of each ablation curve to Eq. (4). 
Table 1 Scattering lengths and fluence thresholds of epithelium and SLP in canine and human samples. Uncertainty is represented as the standard error of the parameters from weighted nonlinear least squares fitting.

\begin{tabular}{|c|c|c|c|c|c|}
\hline Sample & $\ell_{s, \mathrm{ep}}(\mu \mathrm{m})$ & $\ell_{s, \mathrm{SLP}}(\mu \mathrm{m})$ & $F_{\text {th,ep }}\left(\mathrm{J} / \mathrm{cm}^{2}\right)$ & $F_{\mathrm{th}, \mathrm{SLP}}\left(\mathrm{J} / \mathrm{cm}^{2}\right)$ & $z_{\mathrm{ep}}(\mu \mathrm{m})$ \\
\hline Canine VF \#1 & $70.2 \pm 4.2$ & $25.9 \pm 1.2$ & $1.49 \pm 0.05$ & $1.70 \pm 0.17$ & $64.8 \pm 4.8$ \\
\hline Canine VF \#2 & $75.1 \pm 5.6$ & $26.9 \pm 0.5$ & $1.54 \pm 0.06$ & $1.67 \pm 0.15$ & $59.2 \pm 5.0$ \\
\hline Canine VF \#3 & $71.3 \pm 6.6$ & $25.5 \pm 0.6$ & $1.51 \pm 0.06$ & $1.30 \pm 0.12$ & $54.4 \pm 3.7$ \\
\hline Canine VF \#4 & $75.4 \pm 4.1$ & $25.2 \pm 1.7$ & $1.67 \pm 0.06$ & $1.25 \pm 0.13$ & $52.0 \pm 2.2$ \\
\hline Canine VF \#5 & $75.6 \pm 5.6$ & $27.1 \pm 1.6$ & $1.62 \pm 0.06$ & $1.83 \pm 0.22$ & $62.4 \pm 6.3$ \\
\hline Canine VF \#6 & $77.6 \pm 8.6$ & $25.9 \pm 1.4$ & $1.60 \pm 0.08$ & $1.58 \pm 0.19$ & $58.4 \pm 5.0$ \\
\hline Canine VF \#7 & $72.1 \pm 2.9$ & $25.9 \pm 1.3$ & $1.53 \pm 0.04$ & $1.49 \pm 0.11$ & $58.4 \pm 2.8$ \\
\hline Canine VF \#8 & $84.9 \pm 6.2$ & $26.7 \pm 0.9$ & $1.68 \pm 0.06$ & $1.57 \pm 0.12$ & $55.6 \pm 3.4$ \\
\hline Average & $75.3 \pm 5.7$ & $26.1 \pm 1.2$ & $1.58 \pm 0.06$ & $1.55 \pm 0.17$ & $58.2 \pm 4.3$ \\
\hline Human VF \#1 & $44.5 \pm 3.8$ & - & $1.61 \pm 0.07$ & - & $67.2 \pm 5.2$ \\
\hline Human VF \#2 & $40.6 \pm 4.6$ & - & $1.57 \pm 0.09$ & - & $62.0 \pm 4.0$ \\
\hline Human VF \#3 & $42.1 \pm 2.8$ & - & $1.69 \pm 0.15$ & - & $54.6 \pm 2.6$ \\
\hline Human VF \#4 & $43.8 \pm 2.4$ & - & $1.67 \pm 0.08$ & - & $51.3 \pm 4.2$ \\
\hline Human VF \#5 & $42.8 \pm 2.3$ & - & $1.74 \pm 0.08$ & - & $58.8 \pm 2.4$ \\
\hline Average & $42.8 \pm 3.3$ & - & $1.66 \pm 0.10$ & - & $58.8 \pm 3.8$ \\
\hline
\end{tabular}

packed collagen fibrils of the SLP. We measured $z_{\text {ep }}$ in three areas on each sample near ablation data collection ROIs. The presence of two distinct tissue layers is substantiated by our ablation threshold data, where we observed an abrupt increase in the pulse energies required to initiate ablation near $z_{\mathrm{ep}}$, indicating a medium change. Scattering is likely lower in the epithelium due to sparse distribution of cell nuclei and other subcellular scatterers, whereas tightly packed collagen and elastin fibrils distributed throughout the SLP are highly scattering, leading to increased surface pulse energies required to initiate ablation. Further, uncertainty is higher in $E_{\text {th.surf }}$ determined for epithelium than it is in $E_{\text {th,surf }}$ of the SLP. This can be explained by regularly distributed fibrous protein networks in SLP that help to maintain tissue structure postablation, making it easier to determine percentage of FOV removed and thus $E_{\mathrm{th} \text {,surf }}$ with higher certainty.

Ablation curves follow the fit defined by Eq. (4) very well, and small step increases in pulse energy (typically 3 and $8 \mathrm{~nJ}$ increments for epithelium and SLP, respectively) near the point of $50 \%$ ablation helped to achieve a well-defined threshold. Although low variance was observed in scattering properties within species, there was a noticeable difference between the scattering lengths of human and canine tissue. We obtained an average canine $\ell_{s, \mathrm{p}}=75.3 \pm 5.7 \mu \mathrm{m}$ and an average human $\ell_{s, \mathrm{ep}}=42.8 \pm 3.3 \mu \mathrm{m}$. The shorter scattering length of human VF limited experiments to the epithelial layers, as the pulse energies required to ablate at deeper depths exceeded the available laser power.
In general, the width of the ablation threshold curve $\Delta E$ increased with depth, likely caused by the accumulated influence of tissue inhomogeneity at deeper depths. We found the average fluence threshold for human epithelium $\left(F_{\text {th,ep }}=\right.$ $\left.1.66 \pm 0.10 \mathrm{~J} / \mathrm{cm}^{2}\right)$ to be slightly larger than those for canine epithelium and $\operatorname{SLP}\left(F_{\text {th,ep }}=1.58 \pm 0.06 \mathrm{~J} / \mathrm{cm}^{2}\right.$ and $F_{\text {th,SLP }}=$ $\left.1.55 \pm 0.17 \mathrm{~J} / \mathrm{cm}^{2}\right)$.

\subsection{Porcine Pulse Overlap Study}

We utilized the same experimental methodology presented above on freshly excised inferior porcine VFs to study how varying the number of overlapping pulses $N$ affects our $\ell_{s}$ and $F_{\text {th }}$ measurements. We tested four different pulse overlap conditions by varying FOV and/or frame rates: $N=301$ $\left(\mathrm{FOV}=40 \times 40 \mu \mathrm{m}^{2}\right.$, frame rate $\left.=1.11 \mathrm{fps}\right), N=75(\mathrm{FOV}=$ $80 \times 80 \mu \mathrm{m}^{2}$, frame rate $\left.=1.11 \mathrm{fps}\right), N=12(\mathrm{FOV}=120 \times$ $120 \mu \mathrm{m}^{2}, \quad$ frame rate $\left.=3.05 \mathrm{fps}\right), \quad$ and $\quad N=4 \quad(\mathrm{FOV}=$ $200 \times 200 \mu \mathrm{m}^{2}$, frame rate $=3.05 \mathrm{fps}$ ). We obtained two porcine VFs from whole porcine trachea purchased from a local slaughterhouse and tested within $6 \mathrm{~h}$ of animal sacrifice. Epithelial thickness was measured in multiple locations along the ROI as done in the human/canine VF study. Four depths were examined in the epithelium for the four different pulse overlap conditions. We tried to use identical pulse energies between pulse overlap conditions to allow for direct comparison of each condition for a given depth.

Table 2 summarizes epithelial scattering lengths and ablation thresholds for two porcine VF samples measured at four 
Table 2 Scattering lengths and ablation threshold results of two porcine VFs for various number of overlapping pulses, $N$. Uncertainty is represented as the standard error of the parameters from weighted nonlinear least squares fitting.

\begin{tabular}{lccccc} 
& \multicolumn{2}{c}{$\ell_{s, \mathrm{ep}}(\mu \mathrm{m})$} & & \multicolumn{2}{c}{$F_{\text {th, } \mathrm{ep}}\left(\mathrm{J} / \mathrm{cm}^{2}\right)$} \\
\cline { 2 - 3 } \cline { 5 - 6 }$N$ & $\# 1$ & $\# 2$ & & $\# 1$ & $\# 2$ \\
\hline 301 & $58.4 \pm 1.9$ & $60.4 \pm 3.2$ & & $1.50 \pm 0.04$ & $1.60 \pm 0.05$ \\
75 & $59.2 \pm 3.8$ & $58.3 \pm 5.6$ & & $1.45 \pm 0.04$ & $1.61 \pm 0.07$ \\
12 & $59.7 \pm 5.9$ & $61.5 \pm 4.7$ & & $1.57 \pm 0.07$ & $1.64 \pm 0.08$ \\
4 & $59.5 \pm 3.8$ & $62.4 \pm 2.5$ & & $1.68 \pm 0.08$ & $1.86 \pm 0.05$ \\
\hline
\end{tabular}

different pulse overlap conditions. We performed analysis of covariance (ANCOVA) using Minitab statistical software to determine if a significant difference existed between regression coefficients (i.e., slope of regression line, $\ell_{s, \mathrm{ep}}^{-1}$ ) and constants (i.e., $y$ intercept, related to $F_{\text {th,ep }}$ ) generated for all four datasets in each sample. The test statistic used for hypothesis testing follows the method outlined by Clogg et al. ${ }^{25}$ where $p$-value $\leq 0.05$ represents significance.
We saw no significant difference in $\ell_{s \text {,ep }}$ measurements for all four pulse overlap conditions, and no significant difference in $F_{\text {th,ep }}$ for $N=301,75$, and 12 . We did see a significant difference in $F_{\text {th,ep }}$ when comparing $N=301(p=0.05)$ and $N=75$ ( $p=0.04$ ) to $N=4$ in the first VF sample, and a significant difference in $F_{\text {th,ep }}$ when comparing $N=301(p=0.01), N=$ $75(p=0.01)$, and $N=12(p=0.04)$ to $N=4$ in the second VF sample. Figure 2(a) displays the data for all four pulse overlap conditions tested on VF sample \#2. The $y$ intercept for $N=$ 4 data is higher on average than it is for $N=301,75$, and 4 , resulting in a larger $F_{\text {th,ep }}$ for $N=4$. On average, the percent FOV ablated was less at $N=4$ for a given input energy and target depth, confirmed by counting pixels below threshold in the ablated areas using MATLAB. Images shown in Fig. 2(b) illustrate this point; a smaller percentage of the FOV is removed during ablation at $N=4$ for identical pulse energies, suggesting that incubation may play a role in lowering the fluence threshold for $N>4$. Importantly, the exponential increase in input energy required for ablation at each depth scales at the same rate for all $N$ values tested, demonstrating that $\ell_{s, \text { ep }}$ may be estimated with high certainty, regardless of the FOV or frame rate employed. We determined an average porcine $\ell_{s \text {, ep }}=59.9 \pm 4.1 \mu \mathrm{m}$, which is within $20 \%$ of our previous findings. ${ }^{19}$
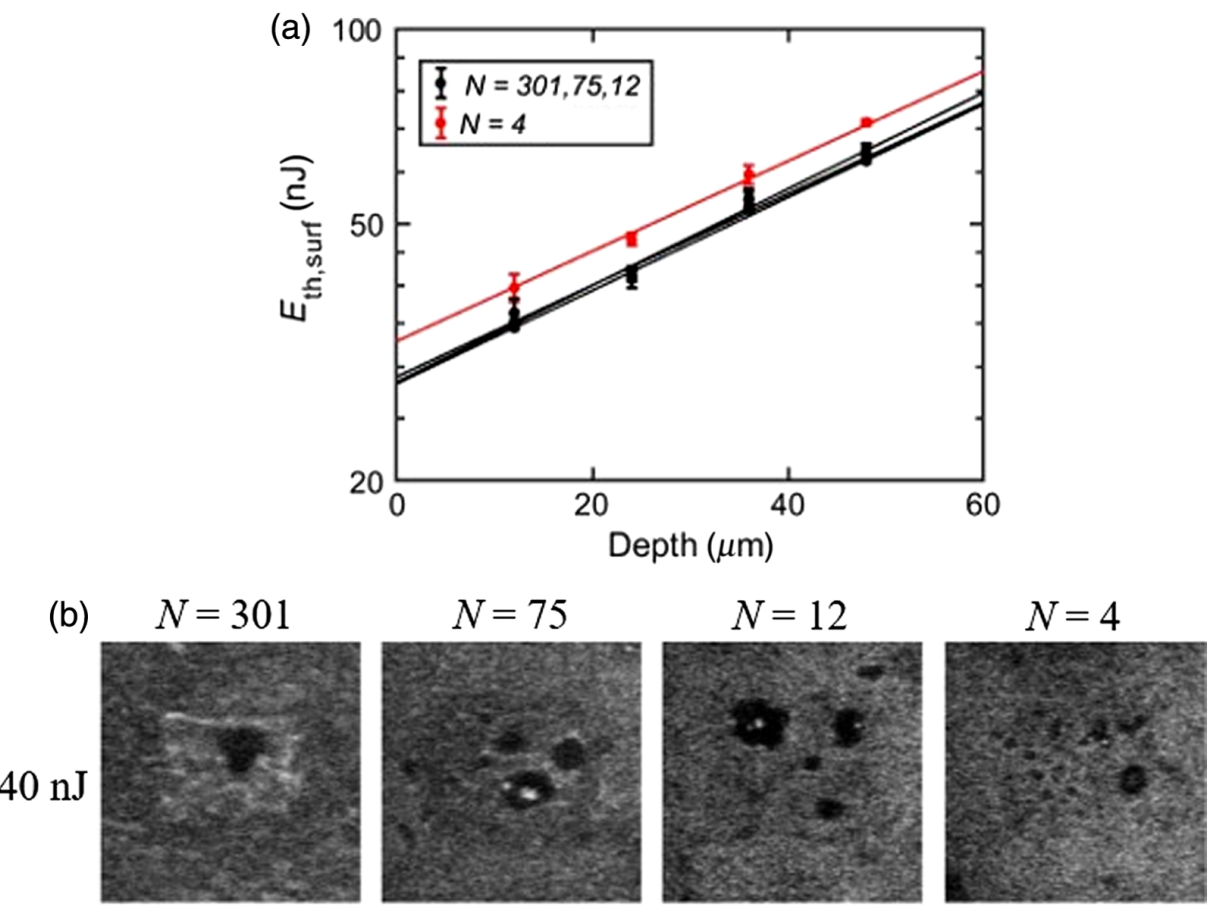

$43 \mathrm{~nJ}$
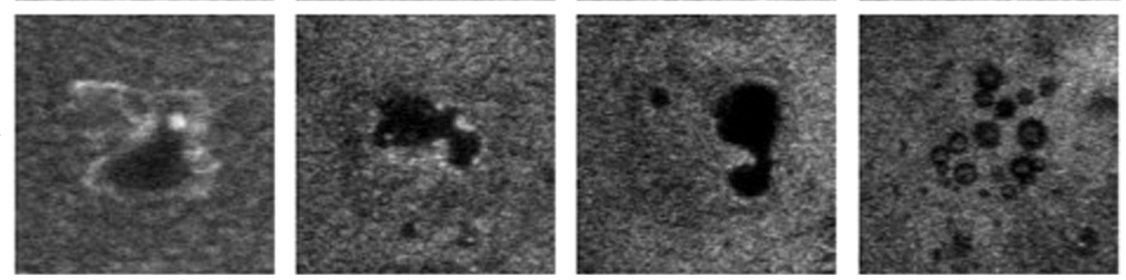

Fig. 2 Cumulative effect results of overlapping number of pulses on $\ell_{s, \mathrm{ep}}$ and $F_{\text {th,ep }}$ determination. (a) The threshold energy at the surface $E_{\text {th.surf }}$ becomes larger at each depth when overlapping pulses decreases to $N=4$ in porcine VF sample \#2, indicating more energy is required to ablate the same percentage FOV with smaller number of pulses. Error bars represent $95 \%$ confidence intervals for the fit of each ablation curve to Eq. (4). (b) Two-photon images after ablation in porcine VF sample \#2 at ablation depth of $20 \mu \mathrm{m}$ for two different pulses energies: $40 \mathrm{~nJ}$ (top row) and $43 \mathrm{~nJ}$ (bottom row). 
The negligible variation in $F_{\text {th,ep }}$ for $N \geq 12$ is in agreement with Rosenfeld et al., ${ }^{26}$ which states that $F_{\text {th }}$ reaches a steady state value for $N>20$ in dielectric materials such as glass where ablation initiates as optical breakdown. In tissue ablation, there is an additional regime of ablation where low-density plasma can lead to ROS-initiated photodamage. ${ }^{27}$ Our irradiance thresholds $\left(1.8 \times 10^{12} \mathrm{~W} / \mathrm{cm}^{2}\right)$ were close to this regime where we would also expect a direct dependence of threshold on the number of overlapping pulses as observed in our earlier findings for laser surgery in severing axons. ${ }^{28}$ Our results, however, do not show as large of dependence on $N$ like Bourgeois et al., which may be due to the less deterministic nature of the highly heterogenous VF tissue architecture. Our results indicate that initiation of ablation at $N \geq 12$ requires $10 \%$ to $15 \%$ less energy than a few pulses, whereas a further increase of $N$ does not offer any significant decrease in $F_{\text {th,ep }}$. We, therefore, propose $N=10$ to 20 to be an optimal number in clinical practice to achieve the desired fast (10 to 20 min per VF) surgical speeds. ${ }^{29}$

\subsection{Storage Condition Study}

To study the effects of tissue storage conditions on scattering length and ablation threshold determination, we measured $\ell_{s, \text { ep }}$ and $F_{\text {th }}$ in fresh porcine VF samples and compared these values to $\ell_{s \text {,ep }}$ and $F_{\text {th }}$ measured in the same samples after storage in PBS. As our human and canine samples were shipped in PBS to prevent dehydration, we deemed it necessary to understand how the measured properties relate to those of native tissue. We extracted VF from whole trachea of freshly sacrificed porcine immediately prior to testing to limit dehydration, then repeated measurements on VFs after $2 \mathrm{~h}$ of storage in PBS at room temperature.

Table 3 summarizes the epithelial scattering lengths and ablation thresholds for two porcine VF samples measured before and after storage in PBS. The ANCOVA test described above showed there was a significant difference between scattering length measurements before and after storage $(p=0.01$, $p=0.05$ for samples 1 and 2, respectively) and a significant difference between the ablation thresholds before and after storage only in sample $2(p=0.02)$. On average, $\ell_{s, \text { ep }}$ is $60 \%$ longer in fresh samples when compared to PBS-stored samples.

Increased scattering in PBS-stored samples may be caused by the larger difference between refractive index of PBS $(n=1.333)$ and refractive index of the epithelium $(n=1.43$ for human skin at $\lambda=800 \mathrm{~nm}$ ) when compared to the refractive index of interstitial fluid $(n=1.365){ }^{30,31}$ Prior work by Genina et $a l .{ }^{32}$ agrees well with our results; they showed scattering coefficients $\left(\ell_{s}^{-1}\right)$ of rat skin increased by $\sim 50 \%$ when stored in an isotonic $0.9 \%$ solution of $\mathrm{NaCl}$. Another

Table 3 Scattering lengths and ablation threshold results of two porcine VFs under different storage conditions. Uncertainty is represented as the standard error of the coefficients and constants from weighted nonlinear least squares fitting.

\begin{tabular}{cccccc} 
& \multicolumn{2}{c}{$\ell_{\text {s,ep }}(\mu \mathrm{m})$} & & \multicolumn{2}{c}{$F_{\text {th }, \text { ep }}\left(\mathrm{J} / \mathrm{cm}^{2}\right)$} \\
\cline { 2 - 3 } \cline { 5 - 6 } & $\# 1$ & $\# 2$ & & $\# 1$ & $\# 2$ \\
\hline Fresh & $101.3 \pm 6.5$ & $99.5 \pm 7.8$ & & $1.64 \pm 0.05$ & $1.83 \pm 0.05$ \\
PBS & $59.8 \pm 3.3$ & $65.7 \pm 6.5$ & & $1.49 \pm 0.05$ & $1.71 \pm 0.08$ \\
\hline
\end{tabular}

confounding factor could be dehydration of tissue samples, which is known to produce an optical clearing effect by reducing the refractive index mismatch between constituent tissue scatterers. ${ }^{33}$ To avoid this effect, we tested samples immediately after excision to mitigate potential dehydration.

These results suggest that scattering length measurements made on PBS-stored human and canine VFs may underestimate the actual scattering lengths of native tissue, as PBS storage is necessary for sample shipment. Hence, we conclude that reduced scattering in intact VF epithelium may lead to lower pulse energies required to ablate tissue within the SLP. This has important implications in surgery, as the lower peak powers required to penetrate epithelium may increase the maximum ablation depth by reducing the probability of unwanted nonlinear effects such as self-focusing, which has been shown to shift the ablation plane toward the surface during deep tissue ablation. ${ }^{34}$

\section{Conclusions}

We determined scattering properties and ablation thresholds of excised human and canine VFs using a nonlinear image-guided, ablation-based method. We found input pulse energies $E_{\mathrm{th}, \text { surf }}$ required to ablate $50 \%$ of the targeted FOV at increasingly deeper depths, fit these data to an exponential curve predicted by Beer's law, and extracted $\ell_{s}$ and $F_{\text {th }}$. We observed low-interspecies variance in scattering properties for both tissue types, yet a substantial difference in $\ell_{s \text {,ep }}$ of human and canine VFs. We studied the cumulative pulse overlap effects on two porcine VF samples and observed no statistically significant change in $F_{\text {th,ep }}$ for $N>12$, and no effect on $\ell_{s, \text { ep }}$ for any pulse overlap tested, providing evidence that this methodology can be applied at a range of spatial scales and scanning rates. Further, we believe surgically relevant speeds are obtainable for $N=10$ to 20, providing lowered ablation thresholds and rapid subsurface void creation. We also studied effects of PBS storage on measurements and determined scattering lengths are 60\% longer in fresh VFs when compared to PBS-stored tissue, suggesting that ablation and subsequent void formation in the SLP may be possible at lower pulse energies in a clinical setting. These results can be used to define an optimal operating regime and predict ablation performance for precise microphonosurgical treatment.

\section{Disclosures}

Authors declare no conflicts of interest.

\section{Acknowledgments}

This work was supported by the National Institutes of Health (NIH) Grant no. R01-DC014783 and the National Science Foundation (NSF) Grant no. CBET-1805998.

\section{References}

1. L. O. Ramig and K. Verdolini, "Treatment efficacy: voice disorders," J. Speech Lang. Hear. Res. 41, S101-S116 (1998).

2. N. Roy et al., "Voice disorders in the general population: prevalence, risk factors, and occupational impact," Laryngoscope 115, 19881995 (2005)

3. S. R. Best and C. Fakhry, "The prevalence, diagnosis, and management of voice disorders in a National Ambulatory Medical Care Survey (NAMCS) cohort," Laryngoscope 121, 150-157 (2011).

4. M. S. Benninger et al., "Vocal fold scarring: current concepts and management," Otolaryngol. Head Neck Surg. 115, 474-482 (1996).

5. S. Hirano et al., "Histologic characterization of human scarred vocal folds," J. Voice 23, 399-407 (2009). 
6. M. S. Hahn et al., "Collagen composite hydrogels for vocal fold lamina propria restoration," Biomaterials 27, 1104-1109 (2006).

7. X. Jia et al., "Hyaluronic acid-based microgels and microgel networks for vocal fold regeneration," Biomacromolecules 7, 3336-3344 (2006).

8. Y. Kishimoto et al., "Chronic vocal fold scar restoration with hepatocyte growth factor hydrogel," Laryngoscope 120, 108-113 (2010).

9. J. K. Hansen et al., "In vivo engineering of the vocal fold extracellular matrix with injectable hyaluronic acid hydrogels: early effects on tissue repair and biomechanics in a Rabbit Model," Ann. Otol. Rhinol. Laryngol. 114, 662-670 (2005).

10. S. Hirano et al., "Prevention of vocal fold scarring by topical injection of hepatocyte growth factor in a Rabbit Model," Laryngoscope 114, 548-556 (2004)

11. L. Hallén, Å. Dahlqvist, and C. Laurent, "Dextranomeres in Hyaluronan (DiHA): a promising substance in treating vocal cord insufficiency," Laryngoscope 108, 393-397 (1998).

12. J. A. Burns et al., "Real-time tracking of vocal fold injections with optical coherence tomography," Laryngoscope 119, 2182-2186 (2009).

13. S. S. Karajanagi et al., "Assessment of canine vocal fold function after injection of a new biomaterial designed to treat phonatory mucosal scarring," Ann. Otol. Rhinol. Laryngol. 120, 175-184 (2011).

14. C. L. Hoy et al., "Towards endoscopic ultrafast laser microsurgery of vocal folds," J. Biomed. Opt. 17(3), 038002 (2012).

15. A. Ben-Yakar et al., "Systems, devices and methods for imaging and surgery," US Patent 9,333,036 B2 (2016).

16. M. Yildirim et al., "Parameters affecting ultrafast laser microsurgery of subepithelial voids for scar treatment in vocal folds," J. Biomed. Opt. 18, 118001 (2013).

17. K. Mahlstedt et al., "An initial assessment of the optical properties of human laryngeal tissue," ORL J. Otorhinolaryngol. Relat. Spec. 63, 372-378 (2001)

18. A. Roggan et al., "The effect of preparation technique on the optical parameters of biological tissue," Appl. Phys. B 69, 445-453 (1999).

19. C. Martin and A. Ben-Yakar, "Determination of scattering properties and damage thresholds in tissue using ultrafast laser ablation" J. Biomed. Opt. 21(11), 115004 (2016).

20. T. J. Farrell, M. S. Patterson, and M. Essenpreis, "Influence of layered tissue architecture on estimates of tissue optical properties obtained from spatially resolved diffuse reflectometry," Appl. Opt. 37, 1958-1972 (1998).

21. J. R. Weber et al., "Noncontact imaging of absorption and scattering in layered tissue using spatially modulated structured light," J. Appl. Phys. 105, 102028 (2009).

22. D. von der Linde and K. Sokolowski-Tinten, "The physical mechanisms of short-pulse laser ablation," Appl. Surf. Sci. 154-155, 1-10 (2000).

23. N. Sanner et al., "Measurement of femtosecond laser-induced damage and ablation thresholds in dielectrics," Appl. Phys. A 94, 889-897 (2009).

24. Q.-T. Nguyen, P. S. Tsai, and D. Kleinfeld, "MPScope: a versatile software suite for multiphoton microscopy," J. Neurosci. Methods 156, $351-359$ (2006).
25. C. C. Clogg, E. Petkova, and A. Haritou, "Statistical methods for comparing regression coefficients between models," Am. J. Sociol. 100, 1261-1293 (1995)

26. A. Rosenfeld et al., "Ultrashort-laser-pulse damage threshold of transparent materials and the role of incubation," Appl. Phys. A 69, S373-S376 (1999).

27. A. Vogel et al., "Mechanisms of femtosecond laser nanosurgery of cells and tissues," Appl. Phys. B 81, 1015-1047 (2005).

28. F. Bourgeois and A. Ben-Yakar, "Femtosecond laser nanoaxotomy properties and their effect on axonal recovery in C. elegans," Opt. Express 15, 8521-8531 (2007).

29. O. Ferhanoglu et al., "A 5-mm piezo-scanning fiber device for high speed ultrafast laser microsurgery," Biomed. Opt. Express 5, 2023 2036 (2014)

30. H. Ding et al., "Refractive indices of human skin tissues at eight wavelengths and estimated dispersion relations between 300 and $1600 \mathrm{~nm}$," Phys. Med. Biol. 51, 1479-1489 (2006).

31. V. Tuchin, Tissue Optics: Light Scattering Methods and Instruments for Medical Diagnosis, SPIE Press, Bellingham, Washington (2015).

32. E. A. Genina et al., "Effect of storage conditions of skin samples on their optical characteristics," Opt. Spectrosc. 107, 934-938 (2009).

33. C. G. Rylander et al., "Dehydration mechanism of optical clearing in tissue," J. Biomed. Opt. 11(4), 041117 (2006).

34. C. Martin and A. Ben-Yakar, "Studying ultrafast laser parameters to deter self-focusing for deep tissue ablation," Proc. SPIE 9740, 97401I (2016)

Liam Andrus received his BS degree in mechanical engineering from the Northern Arizona University in 2013 and his MS degree in biomedical engineering from Texas A\&M University in 2015. He is a graduate student in the Department of Biomedical Engineering at the University of Texas at Austin. He previously worked for Medtronic's Surgical Technologies Division. His research interests include ultrafast laser surgery and nonlinear endoscopy.

Ted Mau received his $\mathrm{PhD}$ in biophysics from UCSF and an MD from Harvard Medical School. He is a professor of otolaryngology-head and neck surgery at the University of Texas, Southwestern Medical Center. He conducts clinical and computational research in the physiology and biomechanics of voice production, as well as in potential therapies for vocal fold scarring. He is a recipient of the American Laryngological Association Casselberry Award.

Adela Ben-Yakar received her $\mathrm{PhD}$ from Stanford University in 2000 and was a postdoctoral-fellow at Stanford and Harvard University in applied physics from 2000 to 2004. She is a professor at the University of Texas at Austin. Her research focuses on microfluidics, ultrafast laser ablation, nonlinear phenomena, and plasmonics. She is a fellow of SPIE, the OSA, and the AIMBE, and the recipient of the NSF Career, Human Frontier Science Program Research, NIH Director's Transformative, and SBI2 President's Innovation awards. 